\title{
Hopf Bifurcation Analysis of a Three-stage-structured Prey-predator System with Multi-delays
}

\author{
Shun-yi Li \\ Department of Mathematics \\ Qiannan Normal College for Nationalities \\ Duyun, Guizhou, 558000, China \\ e-mail: lishunyi19820425@163.com
}

\author{
Wen-wu Liu \\ Department of Mathematics \\ Qiannan Normal College for Nationalities \\ Duyun, Guizhou, 558000, China \\ e-mail: dylww1958@163.com
}

\begin{abstract}
A three-stage-structured prey-predator model with multi-delays is considered. The characteristic equations and local stability of the equilibrium are analyzed, and the conditions for the positive equilibrium occurring Hopf bifurcation are obtained by applying the theorem of Hopf bifurcation. Finally, numerical examples and brief conclusion are given.
\end{abstract}

Keywords-three-stage-structured; prey-predator system; multi-delays; Hopf bifurcation

\section{INTRODUCTION}

In the natural world, there are many species whose individual members have a life history that takes them through two stages|immature and mature. Aiello and Freedman [1] introduced a single-species stage-structured model with time delay in 1990, and they demonstrated the existence and uniqueness of positive equilibrium of the model, which is globally asymptotically stable. Two-stagestructured models have received much attention in the last 20 years [2-4]. In these papers, the authors assume that the life history of each population is divided into distinctive stages: the immature and mature members of the population, where only the mature member can reproduce themselves. However, in the nature many species go through three life stages: immature, mature and old. For example, many female animals lose reproductive ability when they are old.

A three-stage-structured single-species growth model was studied by S.J. Gao [5], the conditions for stability of equilibrium and the sufficient conditions for the existence of a globally asymptotically stable of positive equilibrium of the model are obtained. However, delays play an important role in the dynamics of populations. Naturally occurring complex dynamics are often naturally generated by well formulated delay differential equations (DDE) models. In many processes of the real word, especially, in many biological phenomena, the present dynamics, the present rate of change of the state variables depends not only on the present state of the processes but also on the history of the phenomenon, i. e. on past values of the state variables. A delayed single species with three life history stage and cannibalism model have considered by S.J. Gao [6] and it is shown that the stability of the positive equilibrium can change a finite number of times at most as time delay is increased for some parameter values.

But, there are few papers study the three-stage-structured predator-prey system with delays. A nonautonomous threestage-structured predator-prey system with time delay have considered by S.J. Yang and B. Shi [7], and the existence of a positive periodic solution is obtained, by using the continuation theorem of coincidence degree theory. Recently, a three-stage-structured prey-predator system with predator density dependent is studied by S.Y. Li and X.G. Xue [8], the conditions for the positive equilibrium occurring Hopf bifurcation is obtained and numerical examples are given.

In this paper, we consider following three-stagestructured prey-predator model with multi-delays

$$
\left\{\begin{array}{l}
x_{1}^{\prime}(t)=\alpha x_{2}(t)-\left(\gamma_{1}+\Omega\right) x_{1}(t)-\eta x_{1}^{2}(t)-E x_{1}(t) y(t), \\
x_{2}^{\prime}(t)=\Omega x_{1}(t)-\left(\theta_{1}+a\right) x_{2}(t), \\
x_{3}^{\prime}(t)=a x_{2}(t)-b x_{3}(t), \\
y^{\prime}(t)=k E y(t-\tau) x_{1}(t-\tau)-d y(t)-f y(t) y(t-\tau),
\end{array}\right.
$$

where $x_{i}(t)(i=1,2,3)$ are the densities of immature preys, mature preys and old preys at time $t, y(t)$ is the density of predator at time $t$, respectively. All of the parameters are positive, $\alpha$ is the birth rate of mature population, and $\gamma_{1}, \theta_{1}, b$ are the death rate of immature, mature and old prey population, respectively. $\Omega$ and $a$ are the maturity rate and ageing rate of the prey population, respectively. $\eta$ and $f$ are the density dependent coefficients of immature prey population and predator population, respectively. $k(0<k<1)$ is the rate of conversing prey into predator and $E$ is the predation coefficient. $\tau$ is the desinty dependent delay and gestation delay for predator population.

Note that in (1), the first and the second equations are independent of the third equation, the asymptotic behavior of $x_{3}(t)$ is dependent on that of $x_{2}(t)$. Therefore, we just need to study following subsystem

$$
\left\{\begin{array}{l}
x_{1}^{\prime}(t)=\alpha x_{2}(t)-\gamma x_{1}(t)-\eta x_{1}^{2}(t)-E x_{1}(t) y(t), \\
x_{2}^{\prime}(t)=\Omega x_{1}(t)-\theta x_{2}(t), \\
y^{\prime}(t)=k E y(t-\tau) x_{1}(t-\tau)-d y(t)-f y(t) y(t-\tau),
\end{array}\right.
$$

where $\gamma=\gamma_{1}+\Omega, \theta=\theta_{1}+a$, The initial conditions for (2) are 
$x_{1}(t)=\phi_{1}(t) \geq 0, x_{2}(t)=\phi_{2}(t) \geq 0, y(t)=\phi_{3}(t) \geq 0, t \in[-\tau, 0]$

\section{STBILITY ANALYSIS AND HopF BIFURCATION}

\section{A. Local Stability Analysis}

Obviously, (2) has two boundary equilibrium $E_{0}=(0,0,0), E_{1}\left(x_{1}, x_{2}, 0\right)$ (if condition $C_{1}$ holds), and a unique positive equilibrium $E_{2}\left(x_{1}^{*}, x_{2}^{*}, y^{*}\right)$ (if condition $C_{2}$ holds), where $C_{1}: \alpha \Omega-\gamma \theta>0, C_{2}: k E x_{1}^{*}-d>0$,

$$
\begin{aligned}
& x_{1}=\frac{\alpha \Omega-\gamma \theta}{\eta \theta}, x_{2}=\frac{\Omega}{\theta} x_{1}, \\
& x_{1}^{*}=\frac{f(\alpha \Omega-\gamma \theta)+d E \theta}{\theta\left(k E^{2}+\eta f\right)}, x_{2}^{*}=\frac{\Omega}{\theta} x_{1}^{*}, y^{*}=\frac{k E x_{1}^{*}-d}{f},
\end{aligned}
$$

Let $\bar{E}=\left(\bar{x}_{1}, \bar{x}_{2}, \bar{y}\right)$ be any arbitrary equilibrium. The linearized equations about $\bar{E}$ are

$$
\left(\begin{array}{l}
x_{1}^{\prime}(t) \\
x_{2}^{\prime}(t) \\
y^{\prime}(t)
\end{array}\right)=A\left(\begin{array}{l}
x_{1}(t) \\
x_{2}(t) \\
y(t)
\end{array}\right)+B\left(\begin{array}{c}
x_{1}(t-\tau) \\
x_{2}(t-\tau) \\
y(t-\tau)
\end{array}\right)
$$

where

$$
\begin{aligned}
& A=\left(\begin{array}{ccc}
-\gamma-2 \eta \bar{x}_{1}-\bar{y} E & \alpha & -\bar{x}_{1} E \\
\Omega & -\theta & 0 \\
0 & 0 & -d-\bar{y} f
\end{array}\right), \\
& B=\left(\begin{array}{ccc}
0 & 0 & 0 \\
0 & 0 & 0 \\
k \bar{y} E & 0 & k \bar{x}_{1} E-\bar{y} f
\end{array}\right),
\end{aligned}
$$

and the characteristic equation about $\bar{E}$ is given by

$$
\operatorname{det}\left(A+B e^{-\lambda \tau}-\lambda I\right)=0
$$

(i) From (4), the characteristic equation about $E_{0}$ is given by

$$
\operatorname{det}\left(\begin{array}{ccc}
-\gamma-\lambda & \alpha & 0 \\
\Omega & -\theta-\lambda & 0 \\
0 & 0 & -d-\lambda
\end{array}\right)=0
$$

Namely

$$
(\lambda+d)\left[\lambda^{2}+(\gamma+\theta) \lambda+\gamma \theta-\alpha \Omega\right]=0 .
$$

Then $\lambda_{3}=-d<0$, and $\lambda_{1}, \lambda_{2}$ are the two other roots of $\lambda^{2}+(\gamma+\theta) \lambda+\gamma \theta-\alpha \Omega=0$. By Routh-Hurwitz criterion, $E_{0}$ is local stable if $\gamma \theta>\alpha \Omega$, local unstable if $\gamma \theta<\alpha \Omega$ and $E_{1}$ exist.

(ii) From (4), the characteristic equation about $E_{1}$ is given by

$$
\operatorname{det}\left(\begin{array}{ccc}
-\gamma-2 \eta x_{1}-\lambda & \alpha & -x_{1} E \\
\Omega & -\theta-\lambda & 0 \\
0 & 0 & k E x_{1} e^{-\lambda \tau}-d-\lambda
\end{array}\right)=0,
$$

Namely

$$
\left(\lambda+d-k E x_{1} e^{-\lambda \tau}\right)\left[\lambda^{2}+\left(\gamma+\theta+2 \eta x_{1}\right) \lambda+\theta \eta x_{1}\right]=0 .
$$

Then, $\lambda_{1}, \lambda_{2}$ are the two roots of

$$
\lambda^{2}+\left(\gamma+\theta+2 \eta x_{1}\right) \lambda+\theta \eta x_{1}=0
$$

with negative real parts, and $\lambda_{3}$ is the root of $\lambda+d-k E x_{1} e^{-\lambda \tau}=0$, then $E_{1}$ is local stable if $k E x_{1}<d$, local unstable if $k E x_{1}>d$ and $E_{2}$ exist.

From (i) and (ii), we have the following result.

Theorem 1. (i) $E_{0}$ is local stable if $\gamma \theta>\alpha \Omega$, local unstable if $\gamma \theta<\alpha \Omega$ and $E_{1}$ exist.

(ii) $E_{1}$ is local stable if $k E x_{1}<d$, local unstable if $k E x_{1}>d$ and $E_{2}$ exist.

\section{B. Existence of Hopf Bifurcation}

The characteristic equation about the positive equilibrium $E_{2}$ is given by

$$
\operatorname{det}\left(\begin{array}{ccc}
-\gamma-2 \eta x_{1}^{*}-E y^{*}-\lambda & \alpha & -E x_{1}^{*} \\
\Omega & -\theta-\lambda & 0 \\
k E y^{*} e^{-\lambda \tau} & 0 & \bar{A}-\lambda
\end{array}\right)=0 .
$$

where $\bar{A}=\left(k E x_{1}^{*}-f y^{*}\right) e^{-\lambda \tau}-d-f y^{*}$

Namely

$$
D(\lambda, \tau)=M(\lambda)+N(\lambda) e^{-\lambda \tau}=0
$$

where

$$
\begin{aligned}
& M(\lambda)=\lambda^{3}+m_{2} \lambda^{2}+m_{1} \lambda+m_{0}, N(\lambda)=n_{2} \lambda^{2}+n_{1} \lambda+n_{0}, \\
& m_{2}=\gamma+2 \eta x_{1}^{*}+E y^{*}+\theta, n_{2}=-d, \\
& m_{1}=\left(d+f y^{*}\right)\left(\gamma+2 \eta x_{1}^{*}+E y^{*}\right)+\theta\left(\eta x_{1}^{*}+d+f y^{*}\right), \\
& n_{1}=E f y^{* 2}-d\left(\gamma+\theta+2 \eta x_{1}^{*}\right), m_{0}=\theta \eta x_{1}^{*}\left(d+f y^{*}\right), \\
& n_{0}=\theta x_{1}^{*}\left(k E^{2} y^{*}-d \eta\right) .
\end{aligned}
$$

When $\tau=0$, (5) becomes to

$$
\lambda^{3}+\left(m_{2}+n_{2}\right) \lambda^{2}+\left(m_{1}+n_{1}\right) \lambda+m_{0}+n_{0}=0 .
$$

and

$$
\begin{aligned}
& m_{2}+n_{2}=\gamma+2 \eta x_{1}^{*}+E y^{*}+\theta+f y^{*}>0, \\
& m_{1}+n_{1}=x_{1}^{*}\left(\theta \eta+k E^{2} y^{*}\right)+f y^{*}\left(\gamma+2 \eta x_{1}^{*}+E y^{*}+\theta\right)>0, \\
& m_{0}+n_{0}=\theta x_{1}^{*} y^{*}\left(k E^{2}+f \eta\right)>0,
\end{aligned}
$$

Note that

$$
\begin{aligned}
& \left(m_{2}+n_{2}\right)\left(m_{1}+n_{1}\right)-\left(m_{0}+n_{0}\right) \\
> & \theta\left\{\left[2 \eta f x_{1}^{*} y^{*}+E y^{*}\left(d+2 f y^{*}\right)\right]-\left(f \eta+k E^{2}\right) x_{1}^{*} y^{*}\right\}>0
\end{aligned}
$$

By Routh-Hurwits criterion, all roots of (6) have negative real parts. Then, the equilibrium $E_{2}$ is local stable.

Suppose $\lambda=i \omega(\omega>0)$ is a root of (5) and separating the real and imaginary parts, we have

$$
\left\{\begin{array}{l}
m_{2} \omega^{2}-m_{0}=\left(n_{0}-n_{2} \omega^{2}\right) \cos \omega \tau+n_{1} \omega \sin \omega \tau, \\
\omega^{3}-m_{1} \omega=n_{1} \omega \cos \omega \tau-\left(n_{0}-n_{2} \omega^{2}\right) \sin \omega \tau,
\end{array}\right.
$$

From (7), one can get that

Namely

$$
\left(n_{0}-n_{2} \omega^{2}\right)^{2}+n_{1}^{2} \omega^{2}=\left(m_{2} \omega^{2}-m_{0}\right)^{2}+\left(\omega^{3}-m_{1} \omega\right)^{2} .
$$

where

$$
\omega^{6}+p \omega^{4}+q \omega^{2}+r=0,
$$

$$
\begin{gathered}
p=m_{2}^{2}-2 m_{1}-n_{2}^{2}>0, \\
q=m_{1}^{2}+2 n_{2} n_{0}-n_{1}^{2}-2 m_{2} m_{0},
\end{gathered}
$$


$r=m_{0}^{2}-n_{0}^{2}=\theta x_{1}^{*}\left(m_{0}+n_{0}\right)\left[\eta\left(2 d+f y^{*}\right)-k E^{2} y^{*}\right]$,

If $C_{3}: \eta\left(2 d+f y^{*}\right)<k E^{2} y^{*}$ hold, from (10) we know that (8) has least one positive root . From (7), we have

$$
\cos \omega_{0} \tau=\frac{\left(m_{2} \omega_{0}^{2}-m_{0}\right)\left(n_{0}-n_{2} \omega_{0}^{2}\right)+n_{1} \omega_{0}\left(\omega_{0}^{3}-m_{1} \omega_{0}\right)}{\left(n_{0}-n_{2} \omega_{0}^{2}\right)^{2}+\left(n_{1} \omega_{0}\right)^{2}},
$$

Thus

$$
\begin{aligned}
\tau_{n}= & \frac{1}{\omega_{0}} \cos ^{-1}\left[\frac{\left(m_{2} \omega_{0}^{2}-m_{0}\right)\left(n_{0}-n_{2} \omega_{0}^{2}\right)+n_{1} \omega_{0}\left(\omega_{0}^{3}-m_{1} \omega_{0}\right)}{\left(n_{0}-n_{2} \omega_{0}^{2}\right)^{2}+\left(n_{1} \omega_{0}\right)^{2}}\right] \\
& +\frac{2 n \pi}{\omega_{0}}, n=0,1,2, \cdots
\end{aligned}
$$

Let $\lambda(\tau)=v(\tau)+\mathrm{i} \omega(\tau)$ be the roots of (5) such that when $\tau=\tau_{n}$ satisfying $v\left(\tau_{n}\right)=0$ and $\omega\left(\tau_{n}\right)=\omega_{0}$. We can claim that

$$
\left.\frac{\mathrm{d}(\operatorname{Re} \lambda)}{\mathrm{d} \tau}\right|_{\tau=\tau_{0}}>0 .
$$

In fact, differentiating two sides of (5) with respect to $\tau$, we get

$$
\begin{aligned}
& {\left[\left(3 \lambda^{2}+2 m_{2} \lambda+m_{1}\right)+e^{-\lambda \tau}\left(2 n_{2} \lambda+n_{1}\right)-e^{-\lambda \tau} \tau\left(n_{2} \lambda^{2}+n_{1} \lambda+n_{0}\right)\right]} \\
& =\lambda\left(n_{2} \lambda^{2}+n_{1} \lambda+n_{0}\right) e^{-\lambda \tau} \mathrm{d} \lambda / \mathrm{d} \tau
\end{aligned}
$$

then

$$
\left(\frac{\mathrm{d} \lambda}{\mathrm{d} \tau}\right)^{-1}=\frac{3 \lambda^{2}+2 m_{2} \lambda+m_{1}}{\lambda\left(n_{2} \lambda^{2}+n_{1} \lambda+n_{0}\right) e^{-\lambda \tau}}+\frac{2 n_{2} \lambda+n_{1}}{\lambda\left(n_{2} \lambda^{2}+n_{1} \lambda+n_{0}\right)}-\frac{\tau}{\lambda}
$$

Therefore

$$
\begin{aligned}
& \operatorname{sign}\left[\frac{\mathrm{d}(\operatorname{Re} \lambda)}{\mathrm{d} \tau}\right]_{\lambda=\mathrm{i} \omega_{0}}=\operatorname{sign}\left[\operatorname{Re}\left(\frac{\mathrm{d} \lambda}{\mathrm{d} \tau}\right)^{-1}\right]_{\lambda=\mathrm{i} \omega_{0}} \\
& =\frac{1}{\omega_{0}^{2}} \operatorname{sign}\left\{\operatorname{Re}\left[\begin{array}{l}
\frac{\left(m_{0}+m_{2} \omega_{0}^{2}\right)+2 \omega_{0}^{3} \mathrm{i}}{\left(m_{2} \omega_{0}^{2}-m_{0}\right)+\left(\omega_{0}^{3}-m_{1} \omega_{0}\right) \mathrm{i}} \\
+\frac{n_{2} \omega_{0}^{2}+n_{0}}{\left(n_{0}-n_{2} \omega_{0}^{2}\right)+n_{1} \omega_{0} \mathrm{i}}
\end{array}\right]\right\} \\
& =\frac{1}{\Psi} \operatorname{sign}\left[\begin{array}{l}
\left.\left(m_{2} \omega_{0}^{2}-m_{0}\right)\left(m_{0}+m_{2} \omega_{0}^{2}\right)+2 \omega_{0}^{3}\left(\omega_{0}^{3}-m_{1} \omega_{0}\right)\right] \\
+\left(n_{0}-n_{2} \omega_{0}^{2}\right)\left(n_{0}+n_{2} \omega_{0}^{2}\right)
\end{array}\right] \\
& =\frac{1}{\Psi} \operatorname{sign}\left[2 \omega_{0}^{6}+\left(m_{2}^{2}-2 m_{1}-n_{2}^{2}\right) \omega_{0}^{4}-\left(m_{0}^{2}-n_{0}^{2}\right)\right], \\
& =\frac{1}{\Psi} \operatorname{sign}\left(2 \omega_{0}^{6}+p \omega_{0}^{4}-r\right)
\end{aligned}
$$

where $\Psi=\omega_{0}^{2}\left[\left(n_{0}-n_{2}^{2} \omega_{0}^{2}\right)^{2}+\left(n_{1} \omega_{0}\right)^{2}\right]>0$. Since $p>0$ and $r<0$, then

$$
\operatorname{sign}\left[\frac{\mathrm{d}(\operatorname{Re} \lambda)}{\mathrm{d} \tau}\right]_{\lambda=\mathrm{i} \omega_{0}}=1,\left.\quad \frac{\mathrm{d}(\operatorname{Re} \lambda)}{\mathrm{d} \tau}\right|_{\tau=\tau_{0}}>0,
$$

according to the Hopf bifurcation theorem for functional differential equations [9], we have the following result.

Theorem 2. If $C_{3}: \eta\left(2 d+f y^{*}\right)<k E^{2} y^{*}$ holds, then (i) There exists a $\tau_{0}$, when $\tau \in\left[0, \tau_{0}\right)$ the positive equilibrium $E_{2}$ of (2) is asymptotically stable and unstable when $\tau>\tau_{0}$. (ii) If $C_{3}^{\prime}: \eta\left(2 d+f y^{*}\right)<k E^{2} y^{*}$ and $q>0$ holds, system (2) can undergo a Hopf bifurcation at the positive equilibrium $E_{2}$ when $\tau=\tau_{n}(n=0,1,2, \cdots)$, where $\tau_{n}$ is defined by (11).

Remark 1. It must be pointed out that Theorem 2 can not determine the stability and the direction of bifurcating periodic solutions, that is, the periodic solutions may exists either for $\tau>\tau_{0}$ or for $\tau<\tau_{0}$, near $\tau_{0}$. To determine the stability, direction and other properties of bifurcating periodic solutions, the normal form theory and center manifold argument should be considered [10].

\section{NUMERICAL SIMULATION}

We consider following three-stage-structured system with time delay

$$
\left\{\begin{array}{l}
x_{1}^{\prime}(t)=2.8 x_{2}(t)-1.5 x_{1}(t)-0.25 x_{1}^{2}(t)-2 x_{1}(t) y(t), \\
x_{2}^{\prime}(t)=1.3 x_{1}(t)-0.85 x_{2}(t), \\
y^{\prime}(t)=1.7 y(t-\tau) x_{1}(t-\tau)-0.2 y(t)-0.8 y(t) y(t-\tau),
\end{array}\right.
$$
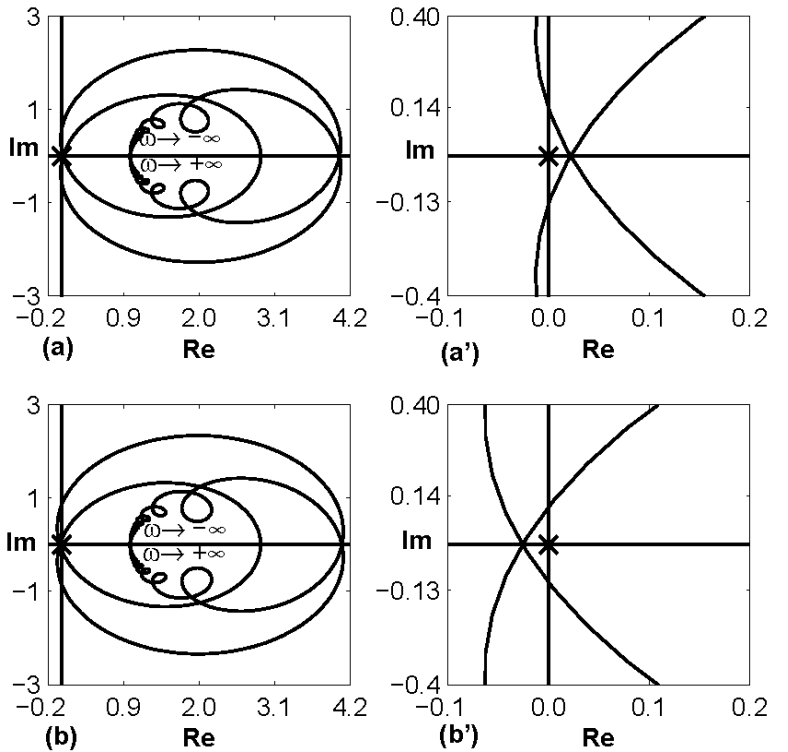

Figure 1. The Nyquist plot of $D(i \omega, 3.7) /(1+i \omega)^{3}$ and $D(i \omega, 3.8) /(1+i \omega)^{3}$, show that positive equilibrium point of (12) are asymptotically stable for $\tau=3.7<\tau_{0}$ ((a) and (a)') and unstable for $\tau=3.8>\tau_{0}$ ((b) and (b’))

where $\quad \alpha=2.8, \gamma_{1}=0.2, \Omega=1.3, \eta=0.25, E=2, \theta_{1}=0.35$, $a=0.5, k=0.85, d=0.2, f=0.8, X(0)=(1,1,1)$. System $(12)$ has unique positive equilibrium point $E_{2}=(0.73,1.12,1.3)$. We evaluate that $p=29.4, r=-7.3, \omega_{0}=0.415, \tau_{0} \approx 3.75$. The the positive equilibrium point $E_{2}$ is asymptotically stable when $\tau=3.7<\tau_{0}$. Because the Nyquist plot [11] of $D(i \omega, 3.7) /(1+i \omega)^{3}$ does not encircle the origin of the complex plane (Fig.1(a) and Zoom around the origin of the Nyquist plot (a')) and the time-series plot are showed (Fig.2 (a)). When $\tau=3.8>\tau_{0}$, the positive equilibrium point $E_{2}$ is unstable. Because the Nyquist plot of $D(i \omega, 3.8) /(1+i \omega)^{3}$ 
encircles the origin of the complex plane (Fig.1(b) and Zoom around the origin of the Nyquist plot (b')) and the Hopf bifurcation occurring around the positive equilibrium $E_{2}$ are shown (Fig.2 (b)). The bifurcating periodic solution (limit cycle) of (12) are stable when $\tau$ from 3.81 to 10 and the amplitudes of period oscillatory are increasing as time delays increased. But, too large time delay would make the population to be die out, because the population very close to zero (Fig.3) as time delay increase to some critical value.
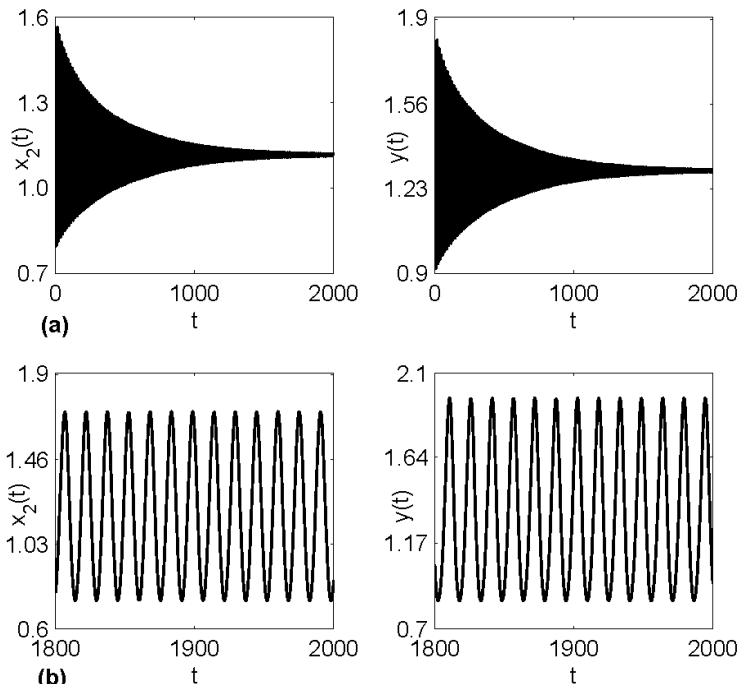

Figure 2. The time-series plot show that positive equilibrium point $E_{2}$ of (12) are: (a) asymptotically stable for $\tau=3.7<\tau_{0}$ and (b) Hopf bifurcation for $\tau=3.8>\tau_{0}$.

\section{CONCLUSION}

In this paper, we consodered a multi-delayed three-stagestructured prey-predator system and analyzed the stability and the characteristic equations of the equilibrium, obtained the conditions of the positive equilibrium occurring Hopf bifurcation. Numerical examples by Nyquist plot and timeseries plot, shown that the system considered local asymptotically stable when $\tau<\tau_{0}$, stable Hopf bifurcation periodic solutions when $\tau>\tau_{0}$ and $\tau$ near $\tau_{0}$. That is to say, time delays can make the positive equilibrium lose stability. It is shown that populations can be coexistence with periodic fluctuating under some conditions and such fluctuation are caused by the time delays. The bifurcating periodic solution (limit cycle) are stable when $\tau$ from 3.81 to 10 and the amplitudes of period oscillatory are increasing as time delays increased. But, too large time delay would make the population to be die out, because the population arbitrary close to zero as time delay increase to some critical value. These are very interesting in mathematics and biology.

\section{ACKNOWLEDGMENT}

This work was supported by the The Natural Science Foundation of Guizhou Province (No. [2011]2116) and Foundation of Key Supported Disciplines of Guizhou province (No. [2009]303).
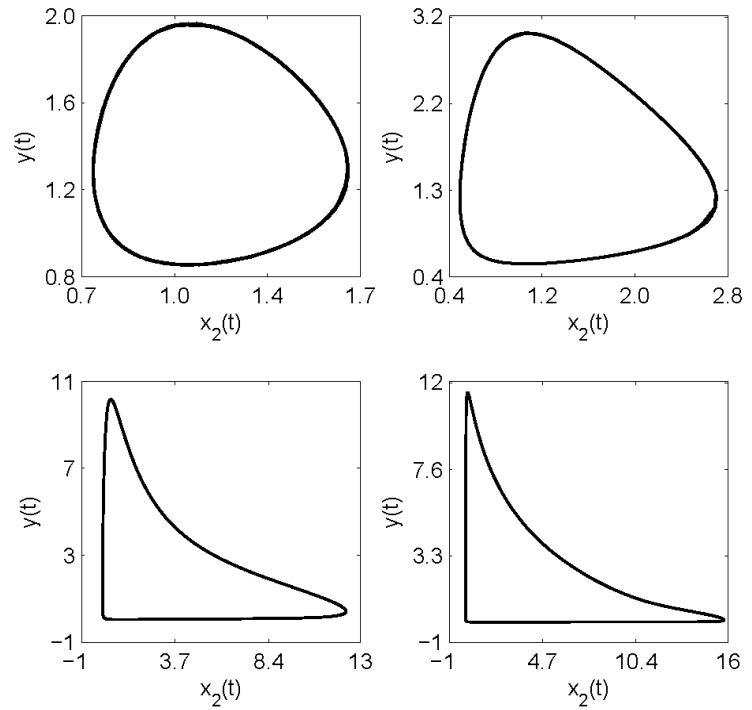

Figure 3. Limit cycles of (12) when $\tau=3.81,4,7,10$.

\section{REFERENCES}

[1] W.G. Aiello and H.I. Freedman, "A time-delay model of singlespecies growth with stage structure,” Math. Biosci., Vol. 101, No. 2, pp. 139-153, 1990.

[2] S.Q. Liu, L.S. Chen and R. Agarwal, "Recent progress on stage structured population dynamics,” Math. Comput. Model., Vol. 36, No. 11-13, pp. 1319-1360, 2002.

[3] S.Y. Li, Z.L. Xiong and X. Wang, "Hopf bifurcation of a two-prey one-predator systemwith time delays,” J. Nanchang Univ. (Engl. Tech.), Vol. 30, No. 1, pp.19-23, 2008. (in Chinese)

[4] S.Y. Li, Z.L. Xiong and R.G. Gu, "A Class of Food Chain System with Stage Structure,” Math. in Prac. and Theo., Vol. 38, No. 13, pp. 102-109, 2008. (in Chinese)

[5] S.J. Gao, "Global Stability of Three-stage-structured Single-species Growth Model,” J. Xinjiang Univ., Vol. 18, No. 12, pp. 154-158, 2001. (in Chinese)

[6] S.J. Gao, "Models for single species with three life history stages and cannibalism,” J. Biomath. Vol. 20, No. 4, pp. 385-391, 2005.

[7] S.J. Yang and B. Shi, "Periodic solution for a three-stage-structured predator-prey system with time delay,” J. Math. Anal. Appl. Vol. 341 No. 1, pp. 287-294, 2008.

[8] S.Y. Li and X.G. Xue, "Hopf bifurcation in a three-stage-structured prey-predator system with predator density dependent," Comm. Comp. Info. Scie., Vol. 288, pp. 740-747, 2012.

[9] J.K. Hale. Theory of Functional Differential Equations. Springer, New York, 1977.

[10] B.D. Hassard, N.D. Kazarinoff and Y.H. Wan. Theory and applications of Hopf bifurcation. Cambridge University Press, Cambridge, 1981.

[11] M.Y. Fu, A.W. Olbrot and M.P. Polis, "Robust stability for timedelay systems: The edge theorem and graphical tests,” IEEE Trans. Autom. Contr., Vol. 34, No. 8, pp. 813-820, 1989. 